\title{
Nachhaltige Beratung
}

\section{Supervision und Coaching sollen vor allem der Qualitätsentwicklung dienen}

Jörg Fellermann und Doris Rosenkranz

Organisationen und Unternehmen, die erfolgreich agieren möchten, brauchen Mitarbeitende, die für Wandel und Krisen gerüstet sind. Unterstützung bieten hierfür Instrumente wie Supervision und Coaching. Wie stark diese Angebote von sozialen Organisationen bereits genutzt werden, untersuchte eine neue Pilotstudie.

Interessenneutrale Beschreibungen des Marktes für Supervision und Coaching sind selten. Im Sommer 2008 wurde im nordbayrischen Regierungsbezirk Unterfranken eine standardisierte und anonyme schriftliche Befragung unter insgesamt eintausend sozialwirtschaftlichen Organisationen und gewerblichen Unternehmen durchgeführt, um Merkmale dieses Marktes zu beschreiben. Die Untersuchung stellt eine Pilotstudie auf der Grundlage einer Zufallsstichprobe dar und erfüllt die Kriterien wissenschaftlicher Validität. Der folgende Beitrag stellt einige Ergebnisse für den Bereich sozialwirtschaftlicher Organisationen vor. (1)

Nahezu 30 Prozent aller befragten Organisationen und Unternehmen nehmen sich die Zeit, den vierseitigen Fragebogen auszufüllen und an das beauftragte Forschungsinstitut zurück zu senden - ein vergleichsweise hoher Wert, zumal mit dem Ausfüllen keinerlei »Incentives « verbunden waren. Befragt wurden Organisationen und Unternehmen aus dem gesamten Sozial- und Bildungsbereich, schulische und vorschulische Einrichtungen ebenso wie Kliniken, Pflegeeinrich-

Jörg Fellermann M.A. ist seit 1997

Geschäftsführer der Deutschen

Gesellschaft für Supervision e. V.

(DGSv) in Köln.

E-Mail joergfellermann@dgsv.de

Prof. Dr. Doris Rosenkranz ist seit 1998

Hochschullehrerin für

Sozialmanagement an der

Fachhochschule Würzburg-Schweinfurt.

E-Mail rosenkranz@fh-wuerzburg.de tungen, Jugendhilfeeinrichtungen, Beratungsstellen sowie Sozial- und Jugendverwaltungen.

Über 80 Prozent der Antwortenden geben auf die Frage, welche Funktion sie in der befragten Organisation einnehmen »Geschäftsführung « oder »Leitung « an. Nimmt man die in größeren Organisationen speziell eingesetzten Personalleiterinnen und Personalleiter hinzu, so ergibt sich eine Quote von nahezu 95 Prozent.

58 Prozent der Befragten geben an, in den letzten drei Jahren ihren Mitarbeiterinnen und Mitarbeitern - vor allem Supervision als Unterstützungsinstrument angeboten zu haben. Dabei geht das Angebot Supervision häufiger mit dem Angebot Coaching einher als mit anderen Unterstützungsleistungen wie Teamentwicklung, Praxisberatung oder Organisationsberatung.

Einrichtungen der psychosozialen Beratung $(82 \%)$ und der Jugendhilfe (87\%), aber auch Einrichtungen zur Hilfe für psychisch kranke $(72 \%)$ oder für behinderte Menschen (55\%) nehmen externe Beratungs- und Unterstützungsleistungen weit mehr in Anspruch als etwa Schulen (31\%) oder vorschulische Einrichtungen (33\%). Diese Spanne ist umso bedeutsamer als etwa in Beratungseinrichtungen nur 69 Prozent der Mitarbeitenden in direktem Kontakt zu Klienten stehen, in Schulen aber über 85 Prozent der Mitarbeitenden direkt mit den Schülern arbeiten.

Gefragt, was den Einsatz von Supervision (und nur von Supervision) veranlasst, antwortet (bei Mehrfachnennungen) nahezu die Hälfte aller Befragten, sie zur Qualitätsentwicklung einzusetzen. Bei »Konflikten « und zur » Weiterqualifikation « von Mitarbeitenden wird Supervision ebenfalls noch $\mathrm{zu}$ jeweils etwa 40 Prozent eingesetzt. Die Unterstützung von Leitungsaufgaben und die Unterstützung der Organisationsentwicklung rangieren mit 28 Prozent rsp. 25 Prozent weiter hinten in der Liste wichtiger Anlässe.
58 Prozent aller sozialwirtschaftlichen Organisationen und Dienste formulieren die Kompetenzverbesserung der Mitarbeitenden als Ziel der Supervision, 46 Prozent die Klärung von Konflikten. Auch die Aufgaben- oder Rollenklärung ist noch für 41 Prozent der Organisationen bedeutsam. Die Verbesserung der Kooperation (30\%), die Erhaltung der Motivation der Mitarbeitenden (28\%) oder die Unterstützung bei der Konzeptentwicklung $(24 \%)$ stehen weniger im Vordergrund (vgl. Abb. 1).

\section{Supervision: Vertrauens- und Beziehungssache}

Bei der Frage, was sie bei der Auswahl von Beraterinnen und Beratern leitet, weisen die Organisationen darauf hin, dass Beziehungen und Empfehlungen wesentliche Kategorien für die Beauftragung sind. So sagen 43 Prozent der Befragten, sie müssten die Beraterinnen und Berater bereits kennen, die sie beauftragen, Empfehlungen aus dem eigenen Haus berücksichtigen 38 Prozent, die Einschätzung eines ratsuchenden Teams ist für 37 Prozent sehr bedeutsam. Auf Listen und Verzeichnisse (von Verbänden, Fachdiensten o. Ä.) greifen noch 15 Prozent zurück, die Internetrecherche gewinnt offenkundig an Bedeutung, fast jeder fünfte Befragte gibt an, das Internet bei der Auswahl zur Hilfe zu nehmen.

\section{Verfügbare Budgets}

Ein gutes Viertel aller befragten Organisationen $(26,5 \%)$ gibt 1.000 bis 3.000 Euro jährlich für Supervision aus, mehr also noch als jenes knappe Drittel, das bis zu 1.000 Euro ausgibt. Gut 14 Prozent der Befragten gibt zwischen 3.000 und 50.000 Euro jährlich für Beratung aus, von dieser letzten Gruppe liegt der größte Teil im Bereich zwischen 3.000 und 5.000 


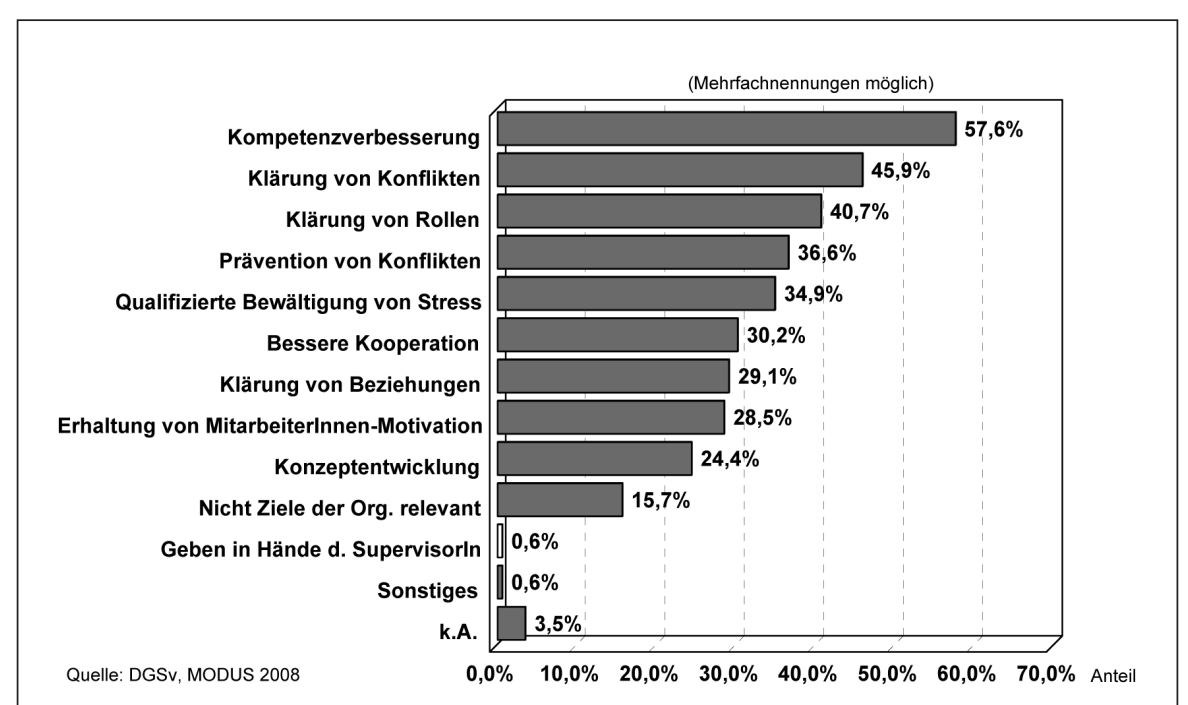

Abb. 1: Eine Kompetenzverbesserung der Mitarbeiterinnen und Mitarbeiter ist das wichtigste Ziel, das mit Supervision erreicht werden soll.

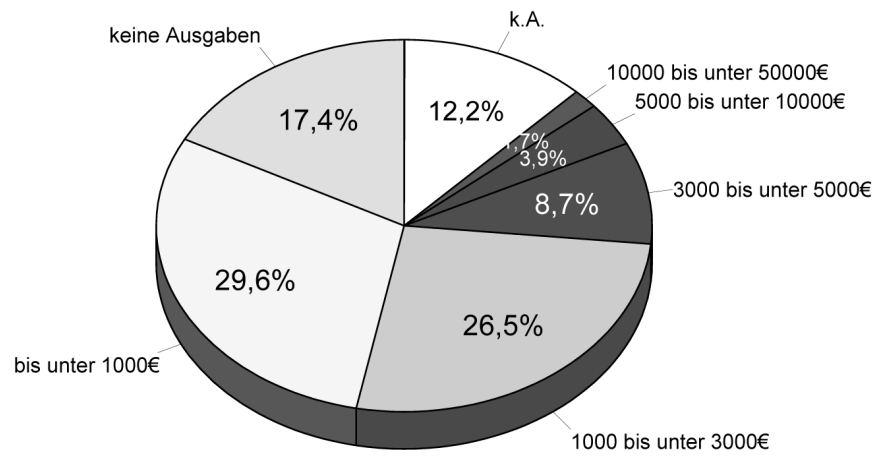

Quelle: DGSv, MODUS 2008

Abb. 2: Mehr als die Hälfte der Organisationen geben für Supervision weniger als 3.000 Euro im Jahr aus.

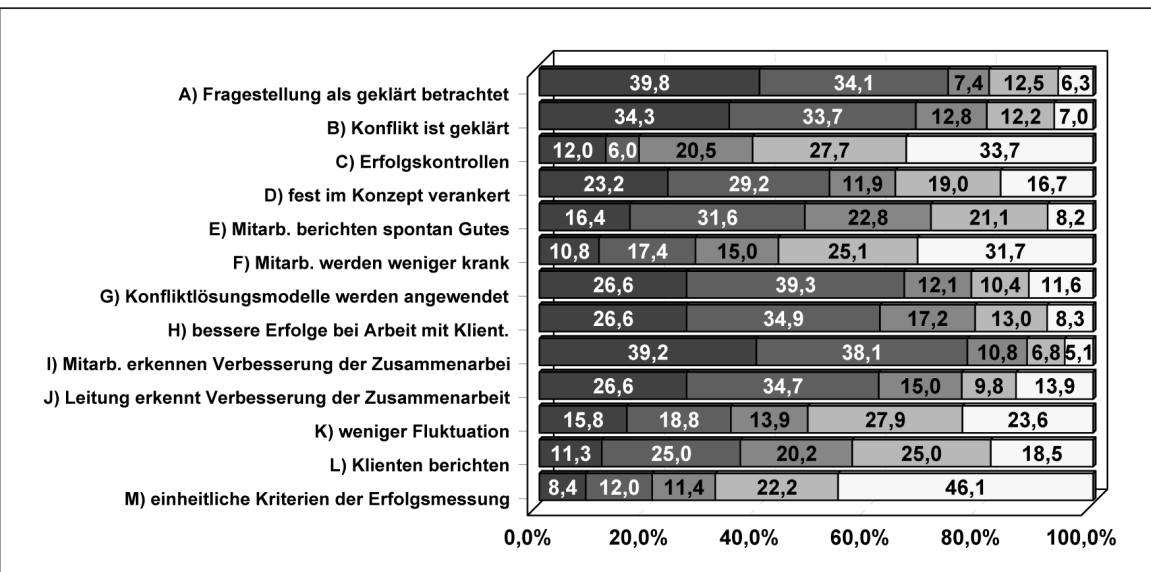

$\square$ Trifft sehr zu $\square$ Trifft eher zu $\square$ Teils/teils $\square$ Trifft eher nicht zu $\square$ Trifft gar nicht zu

Quelle: DGSv, MODUS 2008

Abb. 3: Eine erkennbare Verbesserung der Zusammenarbeit gilt als wichtigstes Erfolgskriterium von Supervision. Allerdings führt weniger als ein Fünftel der befragten Organisationen überhaupt Erfolgskontrollen bei der Supervision durch.
Euro. Immerhin gut 17 Prozent aller Befragten geben nach eigenen Angaben gar nichts für Supervision aus und gut 12 Prozent wollen zur Frage nach den Finanzen keine Antwort geben (vgl. Abb. 2).

\section{Ziele der Unterstützung}

Nahezu 77 Prozent der Organisationen mit einem Budget von mehr als 3.000 Euro investieren das Geld zur Kompetenzverbesserung der Mitarbeitenden durch Supervision, in Häusern mit mittlerem Budget sind es 70 Prozent, in Häusern mit kleinem Budget immer noch fast 55 Prozent. Mittlere Budgets zwischen 1.000 und 3.000 jährlich werden zu 65 Prozent für die Klärung von Konflikten investiert, große Budgets zu 54 Prozent, kleine nur noch zu 43 Prozent. Interessant ist, dass Organisationen mit mittlerem Budget doch immerhin zu 55 Prozent bejahen, Supervision zur Verbesserung der Kooperation einzusetzen.

\section{Erfolgsmessung}

Aufs Ganze gesehen erfährt die Aussage, man stelle einen Erfolg fest, "wenn die Mitarbeiter/innen eine Verbesserung der Zusammenarbeit erkennen « $(77 \%)$, die höchste Zustimmung, gefolgt von der Einschätzung, dass ein Erfolg dann vorliege, »wenn das ... Team ...seine Fragestellung als geklärt « betrachte $(74 \%)$. Wenn »ein Konflikt geklärt « ist oder ein »Konfliktlösungsmodell auch angewendet « wird, sehen noch 68 Prozent rsp. 66 Prozent die Supervision als erfolgreich an. Nur 18 Prozent der Befragten führen nach eigenen Angaben »Erfolgskontrollen « durch, nur 20 Prozent der Befragten bejahen die Frage nach dem Vorliegen von Erfolgskriterien (vgl. Abb. 3).

\section{Ausblick}

Insgesamt zeigt die Studie ein geteiltes Bild: Einerseits wird deutlich, wie groß der Bedarf an Beratungsleistungen ist und wie stark diese Leistungen bereits genutzt werden. Das Management von Beratungsleistungen wird überwiegend im $\mathrm{Be}$ reich der Geschäftsführung und der Personalleitung angesiedelt. Zum anderen zeigt sich, dass die Auswahl von Supervi- 
sorinnen und Supervisoren häufig nicht rein fachlichen Kriterien folgt, sondern über persönliche Kontakte realisiert wird.

Beratung ist Vertrauenssache und damit für standardisierte Formen der Evaluation offenbar bisher weniger zugänglich. (2) Die komplexen Themen, die durch Beratung bearbeitet werden sollen, und der hohe Ressourceneinsatz verlangen Expertise sowohl bei Beratenden wie bei Auftraggebenden. Dies wird besonders deutlich, wenn man die Unsicherheiten in der Beurteilung von Erträgen und Erfolgen in der Beratung betrachtet.

\section{Anmerkungen}

(1) Die Befragung erfolgte durch das Bamberger Wirtschafts- und Sozialforschungsinstitut MODUS, wurde gefördert mit Mitteln der Deutschen Gesellschaft für Supervision e. V. (DGSv), Köln, und wissenschaftlich begleitet durch die Autorin. Bei der Konzipierung der Studie haben Edmund Görtler (Institut MODUS) sowie Christel Baatz-Kolbe, Ulrich Penka, Norbert Scholz und Alois Zang (Supervisorinnen und Supervisoren in Würzburg) verantwortlich mitgewirkt.

\section{"Ziele von Supervision und Coaching sind mehr Kompetenz und weniger Konflikte"}

Präzise Leistungsprofile, kriteriengeleitete Auswahl und Kontraktierung von Beraterinnen und Beratern oder angemessene Evaluationsverfahren - das scheinen wichtige Schritte zu sein, um Beratung als Unterstützung nachhaltiger Organisationsentwicklung $\mathrm{zu}$ sichern und auszubauen. (3)

\section{Die Deutsche Gesellschaft} für Supervision e. V. (DGSv)

ist der 1989 gegründete Berufs- und Fachverband der Supervisorinnen und Supervisoren. 3.600 Mitglieder und 30 Ausbildungsstätten sind hier zusammengeschlossen. Durch standardisierte Ausbildungen und Kontinuierliche Verbesserungsprozesse, durch Forschung und Projektdurchführungen und nicht zuletzt durch das Engagement für gesellschaftliche Themen profiliert die Organisation Beratung in der Arbeitswelt.

Deutsche Gesellschaft für Supervision e. V. (DGSv), Lütticher Straße 1-3, 50674 Köln, Telefon 0221 92004-0, Fax 0221 92004-29, E-Mailinfo@dgsv.de, Internet http://www.dgsv.de
(2) Das beginnt sich zu ändern: Insgesamt 58 wissenschaftliche Arbeiten, die den Nutzen von Supervision untersuchen, stellt der folgende Band zusammen: Deutsche Gesellschaft für Supervision e. V. (Hg.) (2008): Der Nutzen von Supervision. Verzeichnis von Evaluationen und wissenschaftlichen Arbeiten. Kassel university press, Kassel. Kostenfrei downloadbar im Internet: http://www.upress.unikassel.de.

(3) Die Deutschen Gesellschaft für Supervision e. V. bietet kostenfrei ein Info-Paket »Supervision - praktisch eingesetzt « an, formlos zu bestellen per EMail: info@dgsv.de.

\section{Expertenwissen für Führungskräfte}

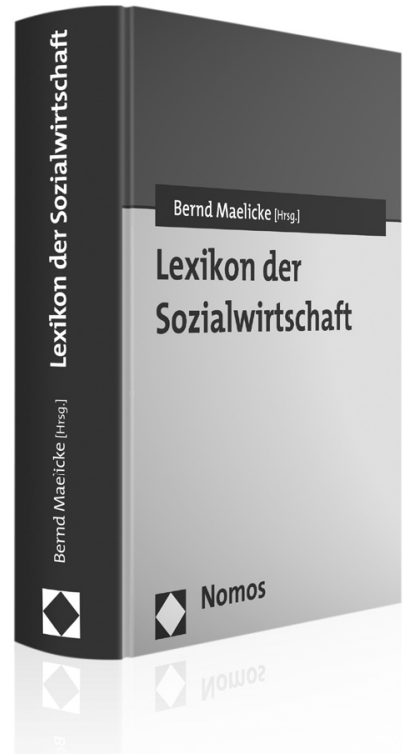

\section{Lexikon der Sozialwirtschaft}

Herausgegeben von Prof. Dr. Bernd Maelicke 2007, 1.128 S., geb., 98,-€, ISBN 978-3-8329-2511-6

"Verdienstvoll und gelungen... Ein qualitätsvolles Handbuch mit Zukunft." Neue Caritas 11/o8

„Es ist das erste Überblickswerk zu der Thematik und stellt auf 1.128 Seiten dar, was gegenwärtig fachlich und sachlich der Stand des Wissens zu sozialwirtschaftlichen Fragen ist."

Prof. Dr. Wolf Rainer Wendt, Zeitschrift für Betreuungsmanagement 69/08

\section{Nomos}

Bitte bestellen Sie im Buchhandel oder versandkostenfrei unter $\downarrow$ www.nomos-shop.de 